\title{
DEEMO: A NEW TECHNOLOGY FOR THE FABRICATION OF MICROSTRUCTURES
}

\author{
J. Elders, H.V. Jansen and M. Elwenspoek \\ MESA Research Institute, University of Twente, P.O. Box 217, NL-7500 AE Enschede, \\ The Netherlands \\ W. Ehrfeld \\ IMM Institute of Microtechnology Mainz, Carl-Zeiss-Strasse 18-20, D-55129 Mainz- \\ Hechtsheim, Germany
}

\begin{abstract}
The recent innovations in dry etching make it a promising technology for the fabrications of micromoulds. The high aspect ratios, directional freedom, low roughness, high etch rates and high selectivity with respect to the mask material allow a versatile fabrication process of micromoulds for subsequent electroplating and embossing, as is demonstrated with the DEEMO process. DEEMO is an English acronym and stands for Dry Etching, Electroplating and MOulding.
\end{abstract}

\section{Introduction}

A new fabrication process for microstructures is described in which the X-ray lithography step of the LIGA process is substituted by a dry etch process. It is demonstrated that using the recent innovations in dry etch processes of silicon or polymers, moulds with variable tapering and high aspect ratios can be fabricated. Dry-etching has considerable advantages over alternative ways of high aspect ratio processing such as UV-lithography, laser ablation, ion milling, spark erosion, and is far cheaper than the LIGA process. The silicon moulds can be used either directly for embossing and moulding or for electroplating and subsequent embossing, as will be explained in the description below of a process we call DEEMO. DEEMO stands for Dry Etching, Electroplating and MOulding. In this paper the feasibility of the DEEMO process is demonstrated for feature sizes of structures of micrometers up to tens of micrometers. Mould inserts have been fabricated by means of dry etching and inserts have successfully been used for the electroplating process and for the embossing process. It is anticipated that while most of the high aspect ratio processing techniques have reached their (theoretical) limit, the continuous development of dry etching and dry etching apparatus will lead to even more flexibility and even better performance of the DEEMO technology in the near future.
High aspect ratio processing

In recent years there has been considerable interest in high aspect ratio processing. Many actuator and sensor principles require high aspect ratios due to the scaling of the physical forces such as electrostatic forces for comb-drive structures [1] and curved electrode actuators [2]. Fluidic devices and chemical analysis systems such as micromachined chromatographs preferably require relatively high aspect ratio structures as well. Although a variety of three dimensional structures such as nozzles, diaphragms and beams have been fabricated in silicon, other materials such as plastics and metals are increasingly studied as well. The availability of other materials increase the applicability of microstructuring in general. For certain applications the availability of different materials is a necessity, such as optical devices directly fabricated in polymers. Inexpensive fabrication processes for microstructuring of different materials are desirable as well. The DEEMO process is able to fulfil these requirements economically.

The LIGA technique can produce extremely high aspect ratio structures by means of irradiating thick resist layers of PMMA with radiation of $0.2 \mathrm{~nm}$ wavelength [3] and subsequent electroplating of the mould insert for moulding or embossing. The costs, however, are high as well, making it economically less attractive. Several techniques have been proposed in order to fabricate economically high aspect ratio structures [4-6]. All of them can be used to directly fabricate the end product, or to fabricate the mould for subsequent replication of the inverse structure of the mould. When these high aspect ratio processing techniques are discussed, the following parameters are of interest: geometrical freedom in two dimensions and in three dimensions, batch- or single structure processing, the maximum aspect ratio (as function of the diameter or width of the structure), the anisotropy, the roughness of the resulting structure, the resolution and the accuracy of the desired image/structure. The term "aspect ratio" is defined as follows: the aspect 
ratio is the ratio of the diameter with the depth of the structure. It should not be possible to observe an angle of the structure with the surface-normal, thus the anisotropy is maximal. Control of the angle is desirable as well. Trenches will be defined as positive aspect ratios, while needles are denoted by means of negative aspect ratios. Typical dimensions of an example structure are a diameter of 5 micrometer and a depth of up to 100 micrometer.

The following techniques will be briefly discussed: UV-lithography, LIGA, ion beam etching, laser ablation, spark erosion and, last but not least, dry etching.

In a (110) silicon wafer highly anisotropic structures with high aspect ratios can be fabricated by means of wet chemical etching in the (100) direction. However, anisotropic wet chemical etching of silicon is not discussed because the profile is controlled by the crystal orientation and thus lacks geometrical freedom in two dimensions, although development in anodic wet etching may alter this situation. As is demonstrated, taking into account the above-mentioned criteria, dry etching has the best potential of economical high aspect ratio processing.

The most well known technique is UV lithography in thick resist layers, followed by subsequent electroplating and if desired, embossing or micromoulding [4]. An advantage of this technique is the fact that an enormous amount of resist and lithography development has been (and will be) performed due to the interest of the IC industry. The image of the technique is determined by the IC compatible mask making technology and lithography. This technique allows geometrical freedom in two dimensions with ever-improving resolutions of the image structure. The roughness is low compared to most of the other techniques. However, the limitation of this technique is in its aspect ratio, due to diffraction of the UV-light (e.g. $436 \mathrm{~nm})$. The result is a sort of cone of light with an angle with respect to the surfacenormal. In practice, the aspect ratio is limited to about 6.

The aspect ratio can only be improved when decreasing the wavelength significantly, such as when using a synchrotron source as in the LIGA technology. In this case, the aspect ratios as well as anisotropy are extremely high. Besides, the roughness of the resulting structures can be lower than $100 \mathrm{~nm}$. Since the mask making commences with the standard IC masks, the accuracy and resolution is in the same order of magnitude as with IC processing. The substrate can be tilted, allowing some geometrical freedom in the third dimension.

An ion beam can be used to ablate material from a substrate and can therefore be used to fabricate anisotropic structures as well. When using IC compatible deposited masks, the characteristics are partly similar to those of UV-lithography. Besides tilting or rotating the substrate with respect to the ion beam source, there is no freedom in the third dimension. Generally only positively tapered profiles and low etch rates are obtained. In addition, due to the low selectivity of an ion beam for the mask material with regard to the ablated material, the aspect ratios are directly related to this selectivity. In practice, aspect ratios will therefore not be very high.

Material can be sputtered away by means of laser ablation as well [6]. This can be done by using a mask, just as with UV-lithography, or by using optics in order to focus the laser beam. When using a mask for the image, the improvement with regard to the aspect ratio due to the shorter wavelength (when using excimer laser with e.g. ArF as laser medium, the resulting wavelength is $193 \mathrm{~nm}$ ) is not very large. An advantage of using optics and moving the focus point is the possibility of real three-dimensional processing. When using optics in order to focus the laser beam, there will be a trade-off between the depth of focus and the resolution. The smallest spot size is in the order of a micrometer, and the depth of focus is in the same order of magnitude. Anisotropic structures become more difficult to fabricate at a small depth of focus. In addition, the use of laser beams does not allow batch processing.

Techniques originating from fine mechanics such as spark erosion are becoming more sophisticated as well, and may also be used for the fabrication of a mould for further replication processes. In the near future it will be possible to fabricate structures of metals with submicron resolution and with roughness below $300 \mathrm{~nm}$ Of course, the spark erosion process is not a batch process.

Recent innovative developments in dry etching using e.g. the $\mathrm{SF} 6 / \mathrm{O} 2 / \mathrm{CHF}_{3}$ gas mixture at relatively high pressure resulted in the possibility of etching silicon with high selectivity with regard to masking material (up to even more than 1000), high etch rates (up to several $\mu \mathrm{m} / \mathrm{min}$.), with directional freedom, and, most important, resulted in control over the etch profile, i.e. ranging from isotropic, negative tapering, vertical walls up to positive tapering. Above all, in silicon, positive aspect ratios of over 20 are feasible and negative aspect ratios above 50 are feasible, and in polymer, positive aspect ratios of over 40 are feasible [7]. The accuracy and resolution are determined by the standard IC mask-making processes. Roughness lower than $50 \mathrm{~nm}$ have been observed. Some geometrical freedom in the third dimension is possible facilitating further replication processes. In addition, the fabrication of dry etched mould inserts can be used for 
electroplating and for e.g. embossing. Therefore, the use of dry etching for the fabrication of mould inserts is very promising.

Sidewall bowing, feature size dependent profiles and RIE lag as well as loading and microloading effects complicate the applicability of dry etching. However, proper control of the parameter setting diminishes the observed effects. In addition, design rules for the microstructures may overcome these effects for the aspect ratios mentioned here. It is anticipated that aspect ratio dependent etching will limit the aspect ratios, although at this moment it is unclear where and what this limit will be.

Since the development of dry etching process and dry etching apparatus continues, further improvements of the process are expected. The results presented here demonstrate that dry etching is promising for the fabrication of silicon micromachined structures as well as for the fabrication of mould insert for electroplating and embossing or moulding processes.

\section{Dry Etching}

The dry etching process has been described elsewhere [see e.g. references 7 and 8]. For the experiments described here, an SF6/O2 plasma has been used to etch silicon. The etching of other materials and the use of different plasmas is reported elsewhere [7,8].

A chromium layer of about $50 \mathrm{~nm}$ has been evaporated onto the (100) silicon wafers as masking material. After lithography and patterning the chromium layer, the wafer has been etched in a Reactive Ion Etcher (RIE). Chromium, as a mask material, has several advantages. The selectivity of a chromium mask with silicon is very high, the chromium is not resputtered up to a d.c. bias voltage of $200 \mathrm{eV}$ and since chromium is a conductor, the mask will not be charged during the etch process. Charging could give rise to strong local fields and influence the process in an uncontrollable manner.

The SF6/O2/CHF3 plasma allows elegant control of physical and chemical etching and deposition effects. Therefore, the etched profile can be controlled by adjusting the parameter setting. The deposition of passivating layers at the sidewalls and bottom of the trench is essential, as well as the removal of the bottom passivating layer. SF6 is dissociated into $\mathrm{F}^{*}$ radicals which are responsible for the chemical etching of the silicon by the formation of the volatile SiF4. The molecular oxygen is dissociated under influence of the rf radiation into oxygen radicals which are able to passivate the silicon surface with $\mathrm{SiOxFy}$. The CHF3 is the source of the $\mathrm{CFx}^{+}$ions. These ions etch $\mathrm{SiOxFy}$ physically forming $\mathrm{COxFy}$, implying that only the horizontal SiOxFy planes are etched. Although the
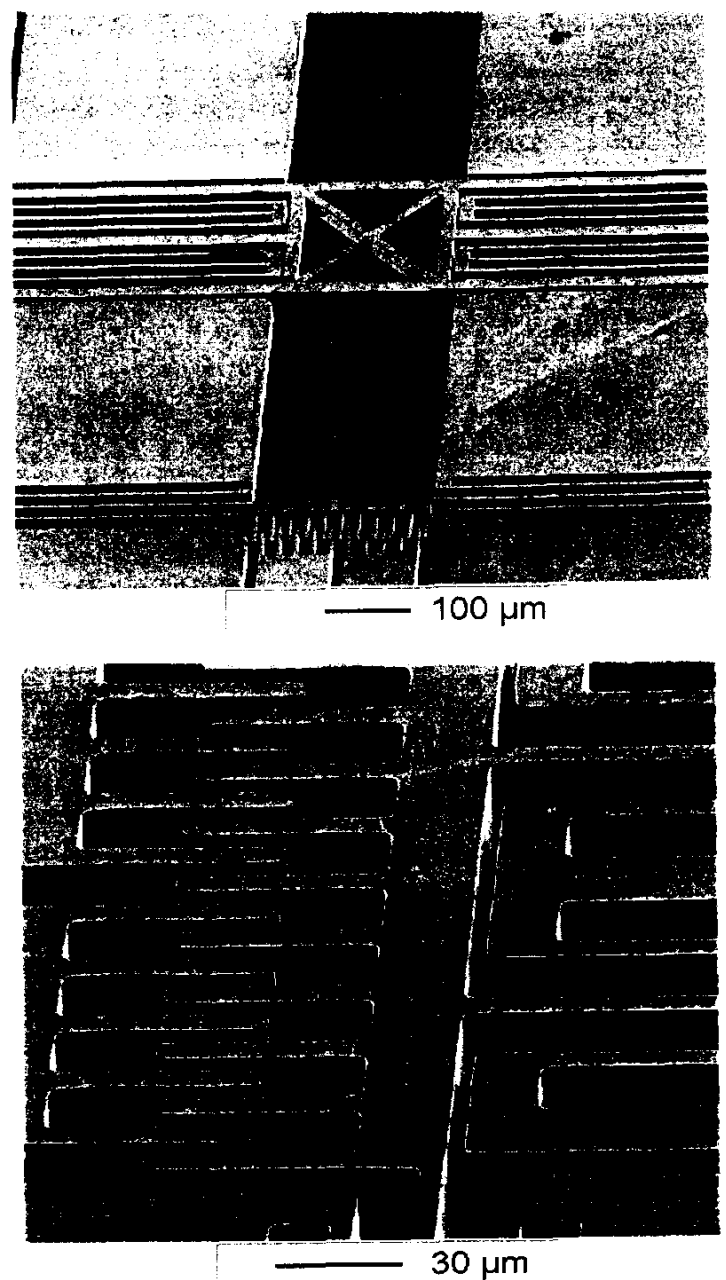

Fig. 1. Scanning Electron Micrographs of a dryetched silicon structure. The etching parameters were: $30 \mathrm{sccm}$ SF6, $5 \mathrm{sccm} \mathrm{O2,} 75 \mathrm{mT}$ Torr, $75 \mathrm{~W}$ and the etch time was $25 \mathrm{~min}$. The etch depth is over $20 \mu \mathrm{m}$. The bar of the top figure indicates 100 micrometer and of the bottom figure 30 micrometer.

$\mathrm{SFx}^{+}$are also able to etch the passivating layer physically by formation of SOxFy, the concentration cannot be independently controlled since it is related to the oxygen concentration, while the $\mathrm{CHF} 3$ concentration can be controlled independently.

As has been mentioned, there is a competition between the etching of the fluorine radicals and the passivation of the oxygen radicals. Increasing the SF6 flow will lead to an increase of the etch rate in all directions resulting in more isotropic profiles. Directional etching can be enhanced when the $\mathrm{CFx}^{+}$content is increased and increasing the oxygen content will decrease the chemical etching component. At high oxygen 
concentrations, positively tapered profiles will be obtained, while at increased CHF3 content the removal of the passivating layer is enhanced, resulting in a less positively tapered profile. In addition, since the ions are positive and since the substrate is negatively biased, it is possible to create negatively tapered profiles due to ion bowing. At even higher CHF3 concentrations, the CFx specimens will scavenge the oxygen radicals and will thus prevent the generation of a passivating layer, resulting in more isotropic profiles. It is evident that with correct control of the pressure, power and flows, high aspect ratios and vertical profiles can be obtained. In figure 1 two Scanning Electron Microscope (SEM) photographs of trenches etched in silicon are shown. The silicon has been etched in a conventional planparallel plate reactor "plasmafab 340" from the STS company. The process parameters are presented in the figure heading. In this case, the roughness of the silicon is lower than $100 \mathrm{~nm}$. The typical feature size of the structures varied from micrometers up to tens of micrometers.

\section{Electroplating}

The silicon trenches can be electroplated in order to fabricate a mould insert. In the case of electroplating, the silicon should be electrically conducting.

For the experiments described here, a nickelsulfamate electrolyte has been used. In the electroplating process, the hydrated nickel ions diffuse towards the silicon structure, the cathode. At the silicon surface, the $\mathrm{Ni}$ ions and electrons combine and hydrogen is formed. There will be a concentration profile of all the reactants and products involved from the cathode (silicon) into the electroplating bath. The process parameters should be selected carefully in order to prevent too much molecular hydrogen evolution which may be incorporated into the Ni structure, or too much stress in the Ni structure or growth induced artefacts of the solid crystal structure. The growth should always be started very slowly, in order to ensure a homogeneous nickel seed layer. A release agent, careful control of the $\mathrm{pH}$, and carefull control of the current is necessary.

In figure 2 two SEM photographs of $\mathrm{Ni}$ structures electroplated from a silicon structure are shown. In this case, $50 \mathrm{~nm} \mathrm{Ti}$ and $100 \mathrm{~nm} \mathrm{Ni}$ have been evaporated on the silicon. The figures demonstrate that successful electroplating on silicon is possible. As can be observed in the figure, the structure is a fine replica of the silicon mould. The process parameters are mentioned in the figure heading.

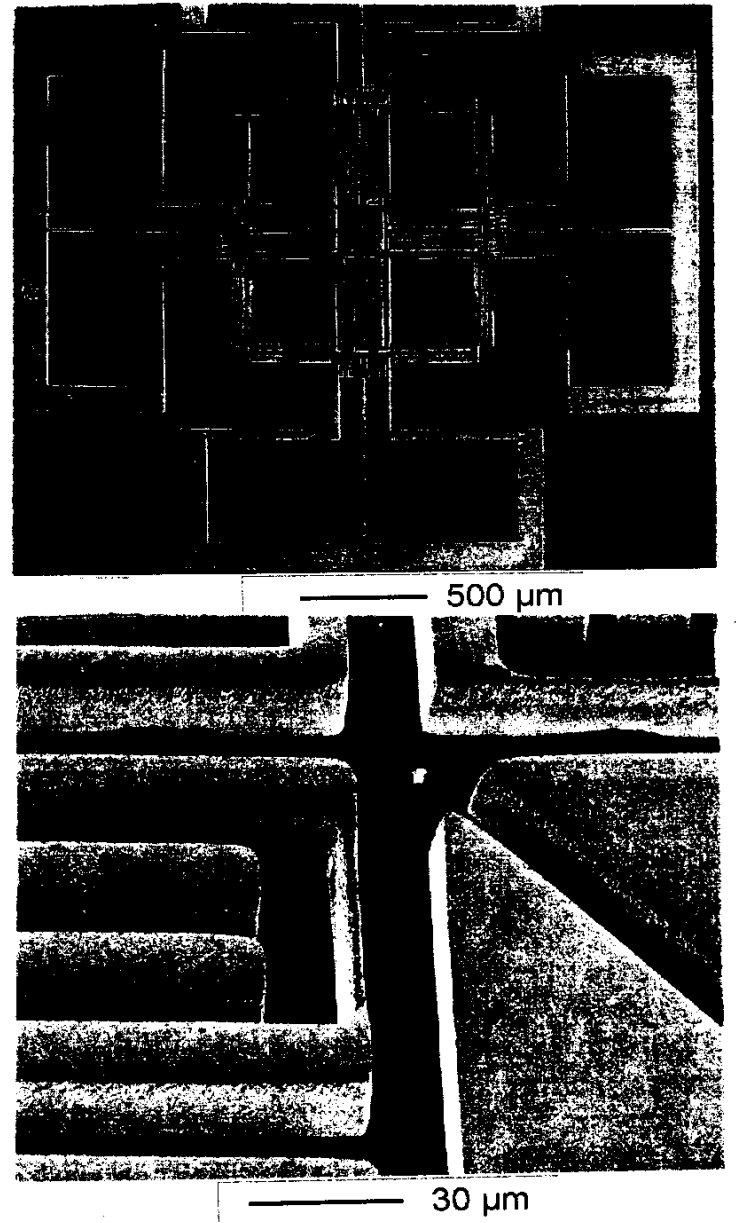

Fig. 2. Scanning Electron Micrographs of a Nickel electroplated structure, using silicon as a mould insert for the electroplating process. The process parameters were: $101.7 \mathrm{~g} / 1 \mathrm{Ni}, 2.92 \mathrm{~g} / \mathrm{l} \mathrm{Br}-40 \mathrm{~g} / 1 \mathrm{H} 3 \mathrm{BO} 3$, releasing agent: $5 \mathrm{ml} / \mathrm{l}, \mathrm{T}=50^{\circ} \mathrm{C}, \mathrm{pH}=3.8$, current density $1 \mathrm{~A} / \mathrm{dm}^{2}, \mathrm{Q}=4.95 \mathrm{~A}$ min. The silicon mould insert has been etched in $\mathrm{KOH}$, which selectively etches silicon with regard to Nickel. Some silicon has been left as a contrast for the $\mathrm{Ni}$, as EDX analysis demonstrated. The bar of the top figure indicates 500 micrometer and of the bottom figure 30 micrometer.

In addition to the above-mentioned experiments, we have demonstrated that direct electroplating on silicon is possible after boron doping of the silicon. Silicon wafers have been boron doped using B2H6 for $1 \mathrm{~h}$ at $1100{ }^{\circ} \mathrm{C}$ resulting in a sheet resistance of $3.9 \Omega / \mathrm{sq}$. These silicon wafers have been electroplated successfully as well. 


\section{Embossing}

The silicon structure has been used as a mould for direct embossing, as is shown in figure 3. For the embossing process, a Collin Press $200 \mathrm{P} / \mathrm{M}$ has been used, allowing a maximum pressure of 250 bar and a maximum temperature of $300^{\circ} \mathrm{C}$. One bar corresponds to a force of $1.2 \mathrm{kN}$. The pressure on the mould insert is the ratio between the applied force and the surface of the mould insert. During the embossing process, the surface may alter due to flowing of the polymer.

The embossing temperature should be high enough in order to heat the polymer material above the glassphase transition temperature and thereby decrease the viscosity of the material substantially, allowing the material to flow. For the polycarbonate sheets of 750 micrometer used for the silicon mould insert experiments, the glass-phase transition temperature was $145^{\circ} \mathrm{C}$. The embossing temperature has been measured in the press-plates which overestimate the temperature of the polymer about $10^{\circ} \mathrm{C}$. Typical embossing temperatures are $185^{\circ} \mathrm{C}-200{ }^{\circ} \mathrm{C}$. When the embossing temperature is too low, the filling of the mould will not be satisfactory and air inclusion may be observed. When the embossing temperature is too high, the flowing of the material may be so abundant that no polymer is left for the microstructures to attach to.

The embossing pressure should be sufficiently high to provide sufficient filling and a critical interplay between the temperalure rising and cooling together with the pressure increase and decrease should ensure proper embossing. Generally, the pressure is applied when the temperature reaches its final value, and will be released just after the cooling started. The pressure cannot be released when the temperature is still high since the material will continue to flow and deformations will be observed. However, the pressure may not be released at too low temperatures since tension will then build up in the microstructures, leading to deformations and cracks in the structures. Process induced stress and thermally-induced stress make the releasing a tedious task. This should be done without tilting the embossed microstructures with respect to the mould insert. The structures in figure 3 have been released by hand, thereby the structures have been tilted as careful inspection reveals. The embossing parameters of the structure in figure 3 are mentioned in the figure heading.

In order to facilitate the releasing of the microstructures, the silicon has been coated with a thin fluorocarbon coating by means of a plasma deposition process. The fluorocarbon coating has been deposited in the same parallel plate reactor (plasmafab 340 from the STS company) in which the structures have been etched [9]. Although quantitative measurements have
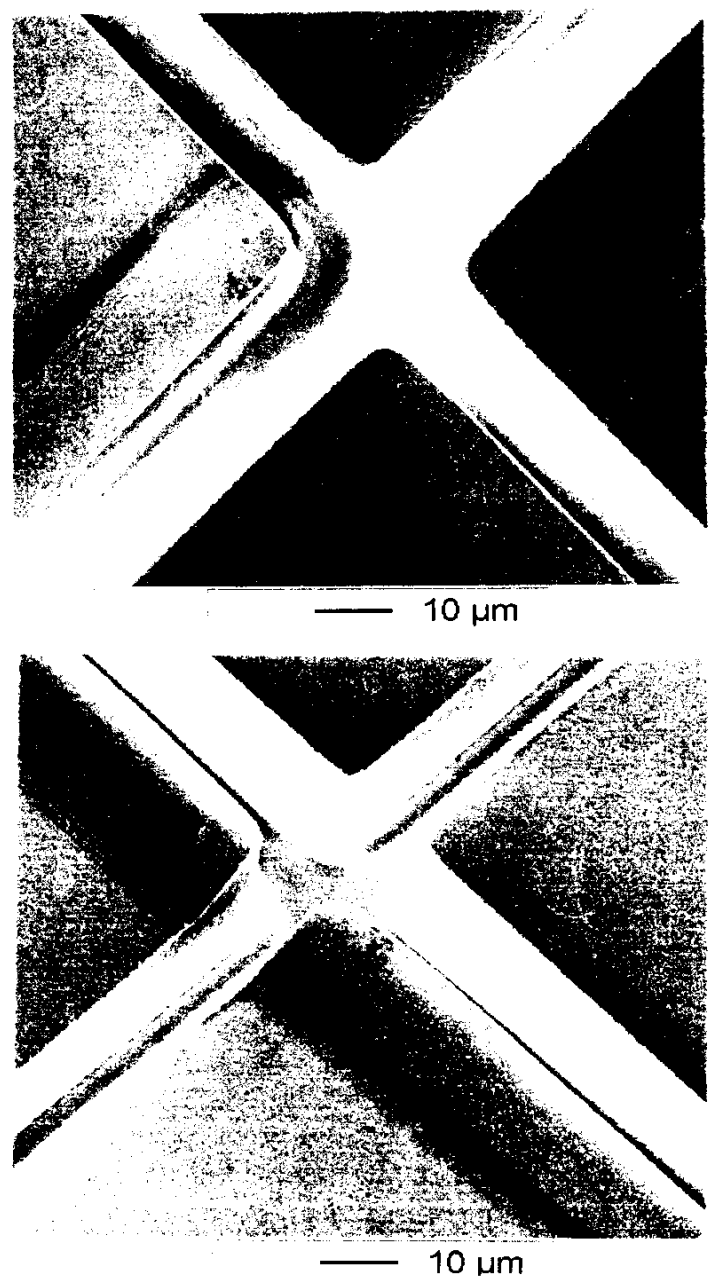

Fig. 3. Scanning Electron Micrographs of embossed polymer structures. Silicon has been used as a mould insert and the polymer is polycarbonate. The process parameters were: $\mathrm{P}=4 \mathrm{bar}, \mathrm{T}=185^{\circ} \mathrm{C}, \mathrm{t}=300 \mathrm{sec}$. The silicon surface was $1 \mathrm{~cm}^{2}$ and the heating cycle has been performed in 300 seconds up to $185^{\circ} \mathrm{C}$, without pressure. The bar of the top and bottom figure indicates 10 micrometer.

not been performed, the fluorocarbon coating did facilitate the releasing of the microstructures.

\section{Discussion}

The dry etch process allows the fabrication of a variety of microstructures, in silicon as well as in polymers. The combination of high aspect ratio processing, control of the angle, high selectivity with respect to the mask material, directional freedom and low roughness allow a versatile fabrication process of moulds, as has 
been demonstrated for the DEEMO process. It has been demonstrated that doped silicon can be electroplated directly and that silicon can be contacted during the electroplating process after deposition of metal layers.

The advantage of direct embossing with silicon, is that the fabrication process of a polymer structure is reduced to silicon etching and embossing facilitating prototyping of plastic microstructures. The difference of the thermal expansion coefficient of polymer and silicon is larger with respect to e.g. nickel. Therefore, higher shear forces are expected when releasing the structure. On the other hand, unlike in the LIGA process, the angle of the mould insert can be controlled by the dry etching process, which facilitates separating the mould from the mould insert.

For most of the high aspect ratio processes the limitations are (theoretically) known. The limitations of the DEEMO process are not yet identified. It is anticipated that with further development of the dry etch process and of plasma sources and plasma reactors, the DEEMO process will become even more valuable compared to other high aspect ratio processes.

\section{Conclusion}

In summary, a new fabrication process, the DEEMO (Dry Etching, Electroplating and MOulding) process has been demonstrated. A variety of mould inserts can be fabricated, either for electroplating processes and/or for moulding and embossing processes. It has been demonstrated that successful electroplating onto doped silicon is possible and that direct embossing using dry etched silicon structures as a mould insert is possible. Above all, the fact that only relatively simple equipment is needed, makes the DEEMO process a promising future trend in micro-electro-mechanical processing, either for silicon structures or for metal and polymer structures.

\section{References}

[1] H.V. Jansen, M. de Boer, R. Legtenberg and M. Elwenspoek, The Black Silicon Method: Proc. Micro Mechanics Europe '94, Pisa, Italy, p. 60. 64.

[2] R. Legtenberg, E. Berenschot, M. Elwenspoek and J.H.J. Fluitman, Electrostatic Curved Electrode Actuators, conf. proc. MEMS '95, January 30-February 2, Amsterdam, The Netherlands.

[3] H. Lehr and W. Ehrfeld, Advanced Microstructure products by Synchrotron radiation Lithography, proc. of the European
Symposium on Frontiers in Science an Technology with Synchrotron Radiation, Aixen-Provence, France, April 5-8, 1994.

[4] L. Paratte, H. Lorenz, R. Luthier, R. Clavel and N.F. de Rooij, Miniature Gear Reduction Unit Driven by a Silicon Electrostatic Wobble Motor, conf. proc. MEMS '94, January 25-28, Oiso, Japan, p. 119-123.

[5] W. Ehrfeld and D. Münchmeyer, Threedimensional Microfabrication using Synchrotron Radiation, Nuclear Instruments and Methods in Physics Research A303 (1991) 523-531.

[6] T.M. Bloomstein and D.J. Ehrlich, LaserChemical Three-Dimensional Writing of Multimaterial Structures for Microelectromechanics, conf. proc. MEMS '91, January 30February 2, Nara, Japan, p. 202-203.

[7] H.V. Jansen, M. de Boer, R. Legtenberg, J. Elders, M. Elwenspoek, J.H.J. Fluitman, EP appl. No. 94202519.8

[8] H.V. Jansen, M. de Boer, J. Burger, R. Legtenberg and M. Elwenspoek, The Black Silicon Method II: Proc. Micro and Nano Engineering 1994, Davos Switzerland, p. 312 313.

[9] J. Elders, H.V. Jansen and M. Elwenspoek, Materials Analysis of Fluorocarbon Films for MEMS Applications, conf. proc. MEMS '94, January 25-28, Oiso, Japan, p. 170-175. 